\title{
A Bluetooth-Based Proximity Sensing AND OBJECT-TRACKING SYSTEM
}

\author{
MikeQu ${ }^{1}, \mathrm{Qi} \mathrm{Lu}^{2}$ and Yu Sun ${ }^{3}$ \\ ${ }^{1}$ Northwood High School, Irvine, CA, 92602 \\ ${ }^{2}$ Department of Social Science \\ University of California, Irvine, CA, 92697 \\ ${ }^{3}$ Department of Computer Science, California State Polytechnic University, \\ Pomona, CA, 91768
}

\begin{abstract}
This paper proposes the concept overall concept of a bluetooth-based proximity IoT device. Its methodology is introduced along with a working prototype. Potential applications and viability for data analysis is also discussed. In summary, such a device accomplishes the goal of closerange information communication in an effective, secure and reliable way through the use of beacons utilizing the BLE (Bluetooth Low Energy) Technology, a variety of different receiver devices that has native support for Bluetooth as well as a database used for data storage and retrieval. The biggest advantage of this technology is that proximity-based Internet has virtually endless potential for applications in the real world, including healthcare, retail and industrial manufacturing.
\end{abstract}

\section{KEYWORDS}

BLE, Proximity sensing, Machine learning

\section{INTRODUCTION}

Throughout history, technology has always been seen as a powerful way to fundamentally transform societies: the industrial revolution in the 1800's, the invention of automobiles, telephones and airplanes had all been historically significant to their respective eras.

The modern society, on the other hand, attributes to one critical new advancement: the invention of the Internet. Unlike many previous inventions, the Internet itself isn't a physical entity but a protocol that allows for the instantaneous communication among various devices located throughout the world, enabling them to send and receive information from one another. As an example, when a person looks up the definition of a word on an online dictionary, that person's computer would establish a connection with an online database and retrieve information from it. The Internet's importance lies in its ability to quickly process and analyze details, improving the general public's ability to access knowledge and materials that were previously beyond their reach [1].

The idea of sharing information among various devices can be similarly applied to the concept of communication within a close proximity [2].This is when the Bluetooth technology come into 
play. Similar to the Internet, Bluetooth can also be used as a medium for interactions among various devices, but with it, data can only be transmitted over short distances. This is an unlikely advantage because due to the Internet's dependence on a series of complex infrastructure requirements, including undersea fiber optic cables, interacting servers as well as its vulnerability from security breaches [3], it is not particularly effective when the two communicating devices are physically close to one another. Bluetooth capabilities, on the other hand, are usually standard on most mobile devices, requiring no additional equipment support, and its ability to send or retrieve data over small distances could cover the gray zone between Internet-based information sharing and face-to-face communication.

Over the past decade, Bluetooth had gradually developed from originally a phone-to-phone data transfer system (Bluetooth 2.0) into a far more sophisticated system that consists of useful features such as a significantly improved battery life, a decrease in discovery time as well as an increase in the speed of data communication [4]. Bluetooth's proximity-based nature, new capabilities, coupled with low-running costs, makes such a device ideal for many tasks that involves data analysis.

\subsection{Open Problem}

In the modern society, many professions incorporate the use of technology to solve problems in their field of study or provide supplemental information for research studies and decision-making. With different adaptations, a Bluetooth-based proximity IoT device can satisfy most of these functions using processes such as crowdsourcing, artificial intelligence learning and so on. This paper will discuss the methodology and a few applications of a Bluetooth-based communication system, what that entails as well as their potential for comprehensive data analysis.

\subsection{Overview of a solution}

The solution to the aforementioned problem involves the use of a device known as an "iBeacon". This device consistently broadcasts a Bluetooth 4.0 signal (BLE) [5] with its pair of two integers that serves as the device's unique identification tag, known as its "major" and "minor". Whereas most bluetooth devices require a process called "pairing" for data transfer, in which the owner of the device has to confirm the exchange on both devices in order for the action to proceed, an iBeacon's communication is one-way, meaning that an iBeacon could transfer information to a receiver device (such as a mobile phone, a laptop etc.) without the need for pairing, making the process significantly simpler [6]. Although the only detail that is actually passed on to the receiver is its identification tags, more information can often be obtained through a variety of techniques, including the use of signal strength, location systems and artificial intelligence.

\section{Detailed Solution}

The solution to the aforementioned problem involves several interacting components ranging from the iBeacon itself, mobile devices, hardware devices, a database, software applications and so on. The functions and contributions of each component to the working system will be presented below. 


\subsection{Qualities of an iBeacon}

Most iBeacons employ a compact Bluetooth Low Energy (BLE) chip, which is programmed to perform all of its predetermined functions and a small coin 3 volt coin battery. Because of these attributes, most beacons are fairly small, approximately five centimeters in diameter and around one centimeter in height. These physical characteristics contribute to another advantage of iBeacons: they are convenient and portable. Furthermore, a typical BLE chip with consistent broadcast intervals of 500 milliseconds consumes very little energy, and requires at most one battery replacement every year, making them extremely reliable under most circumstances [7].

\subsection{Methodology}

As stated above, iBeacons are capable of constantly broadcasting signals that consist solely of its identification tags. Due to native support from various operating systems such as iOS, Android, macOS, Windows and so on, the broadcast signal would eventually reach a device, where, the receiver device will acknowledge the iBeacon's presence by taking in its identification tag and then determining the strength of the accepted signal [8]. These values will eventually be published to a secure database where information can be retrieved for any individual who has permission to access the data at their disposal either through a website or through an OS-specific software. After that, the obtained information would undergo application-specific processes (such as calculations, data analysis) to find the desired outcome to satisfy a predetermined function and the complete content will be displayed on an online console [9]. To address data security, an authentication system is in place where the iBeacon's information will not be displayed or processed unless the correct major and minor are inputted for that device [10].

\subsection{Receivers}

There are two main types of devices capable of receiving information: mobile devices (mobile phones, tablets etc.) and hardware devices (computers). Mobile devices are considered particularly effective when it comes to iBeacon reception, its portability and convenience allows for collected data to be processed virtually anywhere. Hardware devices, on the other hand, are less versatile because they are limited to one single location and requires a consistent power source for it to function [11]. However, when it comes to beacon detection, there is a large difference between the two. In the figure below, hardware receivers can process individual signals at once every 0.3 seconds while mobile receivers is capable of processing requests once every 2 to 3 seconds.

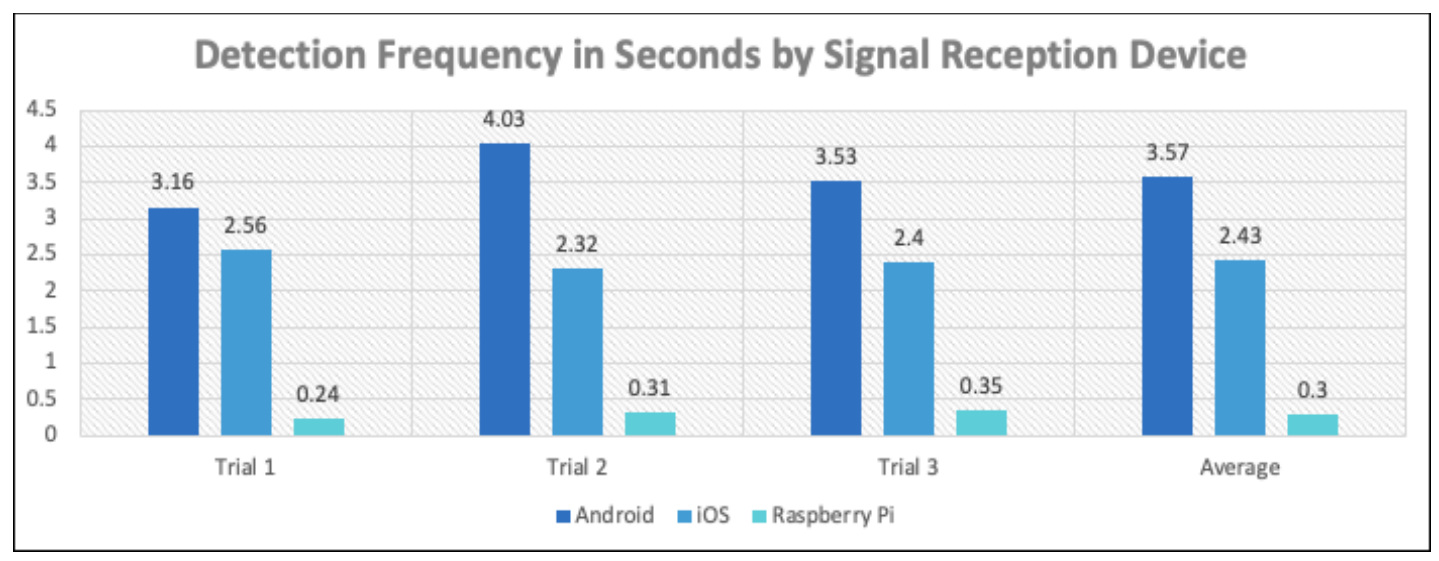


Furthermore, there exists a noticeable range difference among the different types of devices. Shown in the figure below, iOS devices are able to detect beacons from up to 80 meters away, while that range is slightly less for the computer receiver (the Raspberry Pi). Android devices have detection ranges of approximately 50 meters.

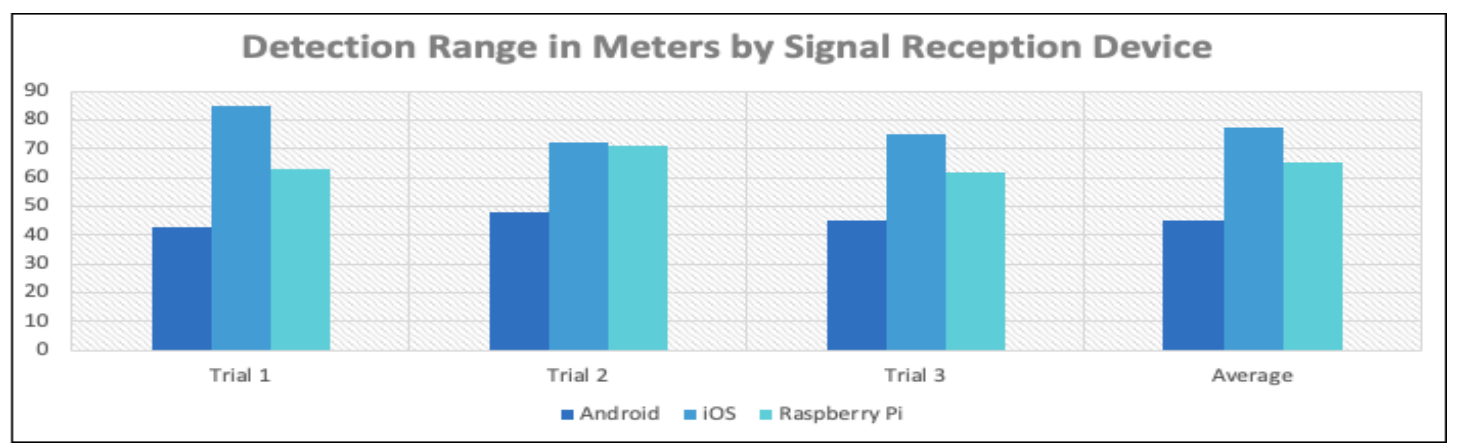

The mobile application consists of three main screens that are intended for authentication, signal reception and application-specific functions respectively. On the authentication page, the major and minor can be inputted to confirm secure access of the iBeacon's details along with the desired nickname of which the iBeacon will go by. On the signal reception page, whether the phone is able to discover an iBeacon signal can be toggled to ensure maximum security. And on the application-unique page, information and analytics needed specifically to serve that specific function are able to be displayed. (In this case a wearable iBeacon used to locate lost pets)

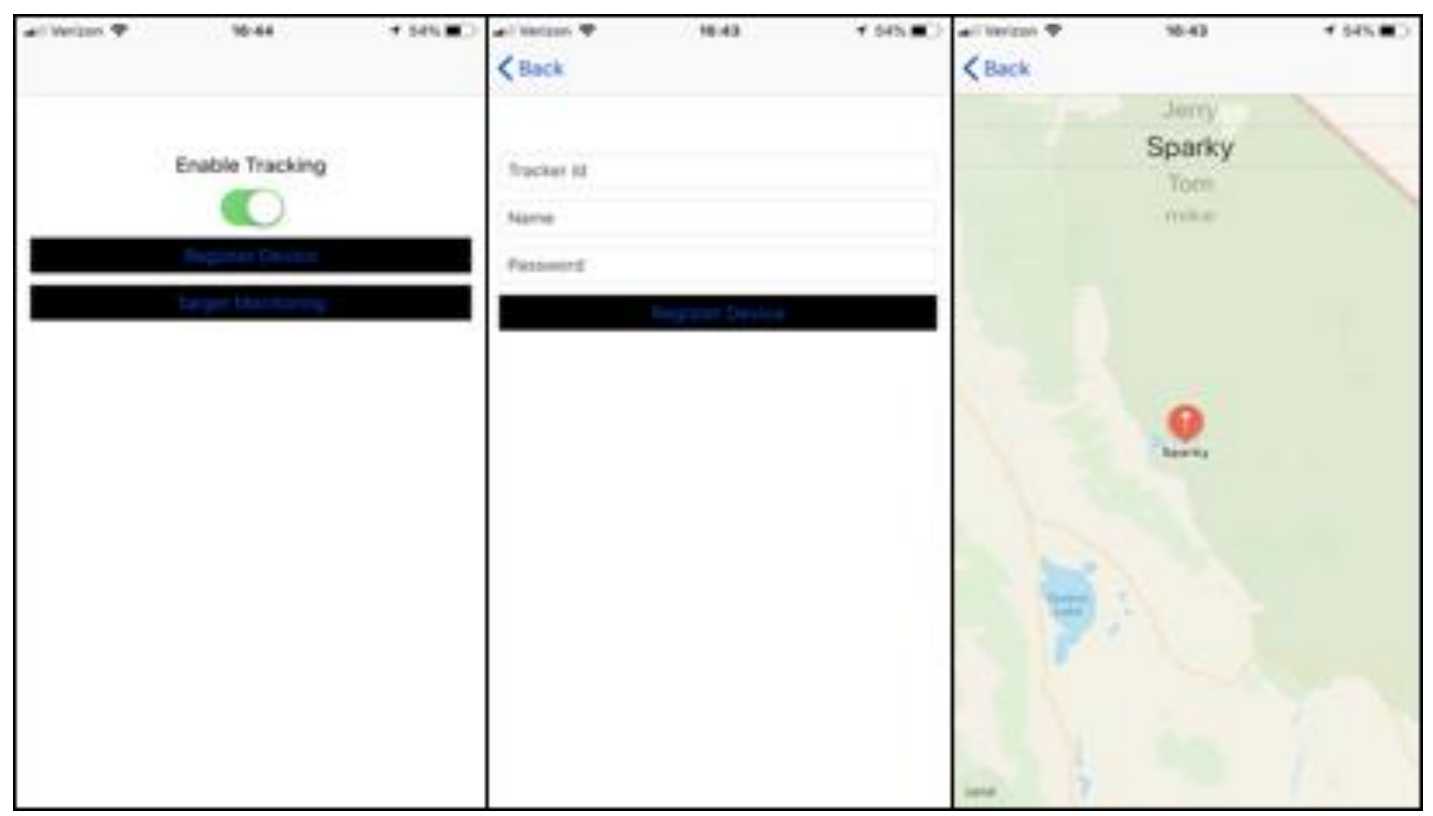

\section{Applications}

As stated before, a proximity-based system has a number of potential applications in real life situations. 


\subsection{Applications in the community}

Although it seems that technology is generally accessible to almost everyone in the society, many individuals are not able to access these products and services due to medical conditions (such as Alzheimer's) or other special circumstances. Because of this, these people can face many notable challenges in the society [12].

This social problem can be solved with the help of Bluetooth beacons. When a receiver device detects a BLE signal broadcasted by a nearby iBeacon, that receiver would log its own location, which, after factoring in signal strength to determine its approximate location respective to the receiver, can represent the approximate location of that iBeacon [13]. This information can then be published to the database and made available for a family member. Along with the iBeacon's reliability and convenience, the concern can be finally alleviated because their whereabouts will be known at all times [14].

\subsection{Applications in research and analytics}

Although there exists research and analytics on a huge variety of different levels ranging all the way from academic studies to scientific research, this application will specifically focus on the business and retail aspect of research and analytics due to its practicality and relevance to real life circumstances. Nowadays, many small businesses use research and analytics as important tools to assess important factors that will contribute to the success of their businesses, such as the popularity of different products, customer tendencies and so on. With the help of Bluetooth beacons, this purpose can be fulfilled easily through the use of iBeacons as a medium for the vendor to interact with his or her customers. In such a case, beacons would be mounted on the walls or next to items of special interest -- or items which the vendor would like promote or gain feedback on. When a customer's receiver device detects a BLE signal broadcasted by such an iBeacon of a certain strength nearby, the receiver device, prompted by this change in signal intensity, would display information that corresponds to or represents that item [15]. If a shopper shows interest and responds to interactive elements (such as buttons, text boxes) about the product on display, specific data would be sent to the vendor, who is then able to make specific decisions to improve his or her business.

\section{DATA}

The advantage of using a proximity-based Bluetooth device mainly lies in its versatility for the obtainment of data and potential within that system for data analysis to reach results and conclusions.

\subsection{Data Collection}

The close-range nature of this system presents it with a great advantage, that the collection of information will involve real people who have established connections with the collector of data (developer to user, vendor to customer etc.). In other words, data collection by this system is typically accomplished with a process known as "crowdsourcing", which is defined as the practice of obtaining relevant and helpful information for a specific cause by engaging with a large group of users [16]. Through crowdsourced data, one is able to make accurate generalizations for specific populations he or she studies, and through making these assumptions 
arrive at more effective and practical results that positively contributes to his or her ability to make changes to his or her cause and achieve a certain goal.

Overall, the collection of data can be done rather simply, as the only essential pieces of data that can be collected with a bluetooth-based proximity beacon are the device's identification tags and the strength of that device's signal. However, because so few pieces of information are collected, the system could be able to be placed anywhere and at anytime, and would still be functional. Although the information obtained is seemingly useless in most situations, they can be used in company with many already-known factors specified by the data collector according to the situation to facilitate data processing [17].

\subsection{Data processing, implementations and results}

The methodologies of data processing can greatly vary according to the goal of data collection. However, as previously stated, a beacon's identification tags and its strength can play an essential role in many cases of data processing.

A beacon's identification tag serves as its unique name, distinguishing itself from other beacons in the same area. This information could be used to establish discrete profiles for data points. Then, through a time interval, each beacon's interactions with receiver devices can be recorded to reveal long-term tendencies. It is possible to obtain generalizations about the data, resulting in the implementation of changes that are relevant to the ultimate goal [18]. For a retail shop, for instance, the vendor will be able to track customer inclinations on various products, by mounting BLE signal-emitting beacons near these products' locations. The information obtained after longterm assessment will be able to reveal a shift in popularity of certain items, allowing the vendor to adjust various business practices such as advertising, pricing and so on to optimize sales and revenue.

On the other hand, the beacon's strength is dependent mostly on the distance between that beacon and its receiver device. If a beacon emits Bluetooth signals that are detected by multiple receiver devices simultaneously at different locations, each receiver device would record a distinct value that represents the signal's strength when it reached that very device. Similarly, when a receiver device takes in Bluetooth signals from multiple beacons, it would match individual signal strengths with that beacon's unique identification tag. Many advantages come out of this sequence of interactions. The biggest benefit being that the practice of crowdsourcing can be successfully accomplished because receivers' common ability to take in multiple different points simultaneously and distinguish them from one another. This is particularly useful in situations that involve the real-time location tracking of a beacon, especially when there are several receiver devices. Suppose the detection radius of each receiver is shown in the figure below as one circle and that there exists one beacon that is constantly emitting bluetooth signals within the system: whether the beacon is detected by only one receiver, or simultaneously detected by two receivers or three receivers can produce information that can effectively narrow down possibilities for the beacon's location. When the beacon's relative strengths experienced by each receiver is taken into a set of calculations, it is completely possible to pinpoint that beacon's exact position [19]. The results can then be used for a variety of purposes, including the drawing of conclusions and further data analysis. 

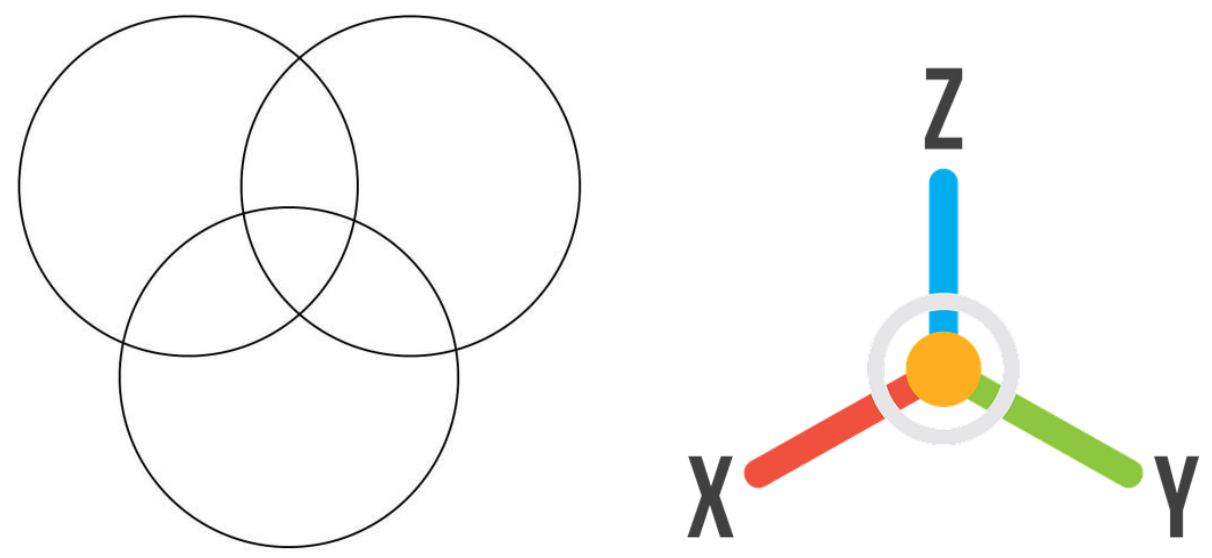

\subsection{Privacy}

The complex interactions that happen among different components of this bluetooth-based proximity IoT system could come with a few risks involving data privacy. Several methods are implemented to protect user data from potential dangers and cybersecurity breaches. As previously mentioned, there exists an authentication process which requires a user to input necessary credentials and identifying information, proving ownership of the beacon before gaining access to data concerning specific beacons. Conversely, the beacon's owner has the option to switch it on or off, and they either agree or deny to give data collectors permission to obtain data using their beacon. It is also possible for receivers to completely reject signals from beacons automatically. Furthermore, beacons are practically anonymous, because only two types of data (I.D. tag and strength) are involved in their operations, neither of which consists of sensitive information [20]. Last but not least, operations involving data always occur after the original data was retrieved from the database, minimizing the information's usefulness in its raw form.

\section{RELATED WORK}

The concept of a "beacon" revolves around a reliable, durable and low-cost device that uses the concept of a proximity-based bluetooth signal emitter to facilitate the process for individuals (such as marketers, certain professionals etc.) to obtain data from their consumers or subjects for the improvement of services or to achieve a specific goal. Specific functions range from real-time location to data collection and analytics can be activated according to individual needs or goals. BLE beacons are especially crucial in this time and age because they are able to overcome various real-life obstacles, including secure, proximity-based data transfer, location-based services, and data collection in general.

\section{CONCluSions AND FUTURE WORK}

This paper proposes the concept overall concept of a bluetooth-based proximity IoT device. Its methodology is introduced along with a working prototype. Potential applications and viability for data analysis is also discussed. In summary, such a device accomplishes the goal of closerange information communication in an effective, secure and reliable way through the use of 
beacons utilizing the BLE (Bluetooth Low Energy) Technology, a variety of different receiver devices that has native support for Bluetooth as well as a database used for data storage and retrieval. The biggest advantage of this technology is that proximity-based Internet has virtually endless potential for applications in the real world, including healthcare, retail and industrial manufacturing.

Future work for this project will involve the actual implementation of the aforementioned prototype. Long term tests on its functionality in real life will be conducted along with efficiency at data transfer.

\section{REFERENCES}

[1] Mattern F., Floerkemeier C. (2010) From the Internet of Computers to the Internet of Things. In: Sachs K., Petrov I., Guerrero P. (eds) From Active Data Management to Event-Based Systems and More. Lecture Notes in Computer Science, vol 6462. Springer, Berlin, Heidelberg

[2] Ashton, K.: That Internet of Things Thing. RFID Journal (2009),

[3] Chakrabarti, A.; Manimaran, G., "Internet infrastructure security: a taxonomy", IEEE Network, Volume: 16 Issue: 6, pp.13-21,Nov/Dec2002

[4] R.A. Rashid, "Bluetooth performance analysis in personal are network (PAN)", Published in RF and Microwave Conference 2006. RFM 2006. International, 12-14 Sept. 2006.

[5] Duquennoy, S., Grimaud, G., Vandewalle, J.-J.: Smews: Smart and Mobile Embedded Web Server. In: Proc. Int. Conf. on Complex, Intelligent and Software Intensive Systems, pp. 571-576 (2009)

[6] Gomez, C.; Oller, J.; Paradells, J. Overview and Evaluation of Bluetooth Low Energy: An Emerging Low-Power Wireless Technology. Sensors 2012, 12, 11734-11753.

[7] Kevin Bouchard, RaminRamezani, ArashNaeim, "Features based proximity localization with Bluetooth emitters", Ubiquitous Computing Electronics \& Mobile Communication Conference (UEMCON) IEEE Annual, pp. 1-5, 2016.

[8] B. G. A. Kumar, K. C. Bhagyalakshmi, K. Lavanya, K. H. Gowranga, "A Bluetooth low energy based beacon system for smart short range surveillance", Proc. IEEE Int. Conf. Recent Trends Electron. Inf. Commun. Technol. (RTEICT), pp. 1181-1184, May 2016.

[9] S. Rajpoot, S. Kumar, P. Singh, "Implementing the physical web using Bluetooth low energy based beacons and a mobile app", theInternational Conference on Innovation and Challenges in Cyber Security (ICICCS-INBUSH), 2016.

[10] "Mobile Authentication System based on National Regulation and NFC Technology", the IEEE FirstInternational Conference on Data Science in Cyberspace, pp. 590-595, 2016.

[11] M. S. Aman, H. Jiang, C. Quint, K. Yelamarthi, A. Abdelgawad, "Reliability evaluation of ibeacon for micro-localization", 2016 IEEE 7th Annual Ubiquitous Computing Electronics Mobile Communication Conference (UEMCON), pp. 1-5, Oct. 2016.

[12] H. H. Strey, P. Richman, R. Rozensky, S. Smith and L. Endee, "Bluetooth low energy technologies for applications in health care: proximity and physiological signals monitors," 2013 10th International Conference and Expo on Emerging Technologies for a Smarter World (CEWIT), Melville, NY, 2013, pp. 1-4. 
[13] Koühne, Markus, and Jürgen Sieck. "Location-based services with iBeacon technology." In Artificial Intelligence, Modelling and Simulation (AIMS), 2014 2nd International Conference on, pp. 315-321. IEEE,2014.

[14] Sehul A. Thakkar, Sunil Patel, Brijesh Kamani, "iBeacon: newly emerged technology for positioning and tracking in indoor place", International Journal of Advanced Research in Computer and Communication Engineering, vol. 5, no. 3, March 2016.

[15] N. Adkar, A. Talele, C. Mundhe and A. Gunjal, "Bluetooth Beacon Applications in Retail Market," 2018 International Conference On Advances in Communication and Computing Technology (ICACCT), Sangamner, 2018, pp. 225-229.

[16] K. M. Gebze, "The positional accuracy of crowdsourced geographic data from open source web GIS," 2017 International Symposium on Geoinformatics (ISyG), Malang, 2017, pp. 77-80.

[17] S. Kajioka, T. Sakuma and I. Takeuchi, "Comparative Sequential Pattern Mining of Human Trajectory Data Collected from a Campus-wide BLE Beacon System," 2019 IEEE International Conference on Pervasive Computing and Communications Workshops (PerCom Workshops), Kyoto, Japan, 2019, pp. 706-711.

[18] J. Weppner and P. Lukowicz, "Bluetooth based collaborative crowd density estimation with mobile phones," 2013 IEEE International Conference on Pervasive Computing and Communications (PerCom), San Diego, CA, 2013, pp. 193-200.

[19] Y. Wang, Q. Yang, G. Zhang and P. Zhang, "Indoor positioning system using Euclidean distance correction algorithm with bluetooth low energy beacon," 2016 International Conference on Internet of Things and Applications (IOTA), Pune, 2016, pp. 243-247.

[20] W. Zhao, T. Qiu and X. Luo, "Automatic User Authentication for Privacy-Aware Human Activity Tracking Using Bluetooth Beacons," 2018 IEEE International Conference on Systems, Man, and Cybernetics (SMC), Miyazaki, Japan, 2018, pp. 3609-3613. 